\title{
THE SUPERSYMMETRY SOFT-BREAKING LAGRANGIAN - WHERE EXPERIMENT AND STRING THEORY MEET
}

\author{
G. L. Kane \\ Randall Laboratory of Physics University of Michigan, Ann Arbor, MI 48109 \\ gkane@umich.edu
}

\begin{abstract}
After an introduction recalling that we expect low energy supersymmetry to be part of our description of nature because of considerable indirect evidence and successful predictions, and a discussion of the essential role of data for formulating and testing string theory, these lectures focus on the role of the supersymmetry soft-breaking Lagrangian in connecting experiment and string theory. How to measure $\tan \beta$ and the soft parameters is examined via a number of applications, and the difficulty of measuring $\tan \beta$ at hadron colliders is explained. In each case the important role of soft phases is made explicit, and the true number of parameters is counted. Applications include the chargino and neutralino sectors, the Higgs sector and how its results change when phases are included, measuring the (relative) gluino phase, $\mathrm{CP}$ violation in $\mathrm{K}$ and $\mathrm{B}$ systems and whether all CP violation can be due to soft phases, how to learn if the LSP is the cold dark matter of the universe, and baryogenesis. It is emphasized that the success of supersymmetry in explaining the breaking of electroweak symmetry is probably its most important achievement, and implications of that explanation for superpartner masses are shown. Combining many of these considerations, a further application argues that a lepton collider of total energy $600 \mathrm{GeV}$ with a polarized beam is one we can be confident will do important physics even after LHC. The question of the origins of CP violation, whether the CKM phase can be zero, and the possibility that the soft phases can tell us about compactification and supersymmetry breaking are discussed. Some of the applications and issues are examined in a D-brane based model that can describe the usual collider and dark matter phenomenology, and includes phases and CP violation as well.
\end{abstract}

\section{INTRODUCTION - SUPERSY- MMETRY? - HOW CAN WE RE- LATE EXPERIMENT AND ST- RING THEORY?}

We are confident the Standard Model of particle physics (SM) will be extended, and its foundations strengthened, because

- it cannot explain the breaking of the electroweak symmetry (EWSB) to accommodate mass

- it cannot explain the baryon asymmetry of the universe

- it cannot provide the cold dark matter of the universe

- it cannot incorporate gravity

- it cannot describe neutrino masses.

There are strong reasons to expect that low energy supersymmetry is the probable outcome of experimental and theoretical progress, and that it will soon be directly experimentally confirmed. By low energy supersymmetry, we mean (softly) broken supersymmetry with an effective soft Lagrangian whose mass parameters are typically of order the electroweak scale but otherwise not special or constrained. It is worthwhile to recall that the main reasons we take low energy supersymmetry very seriously are not its elegance or its likely string theoretical motivations, but its 
successful explanations and predictions. Confidence that low energy supersymmetry is part of the correct description of nature is not a matter of belief; it is based on evidence. Of course, it could happen that all these successes are coincidences, so we must either find superpartners and a light Higgs boson, or demonstrate they do not exist(in which case low energy supersymmetry does not describe nature). The main successes of low energy supersymmetry then are:

- Low energy supersymmetry, with string-motivated boundary conditions (some quark Yukawa couplings such as the top Yukawa coupling of order unity, and no $\mu$ term in the superpotential because the theory describes massless strings), can provide the explanation of how the electroweak symmetry is broken. First, we write the SM effective scalar potential as $V=m_{\phi}^{2} \phi^{2}+\lambda \phi^{4}$, then supersymmetry first requires that $\lambda=\left(g_{1}^{2}+\right.$ $\left.g_{2}^{2}\right) / 2$, where $g_{1}$ is the $\mathrm{U}(1)$ coupling and $g_{2}$ is the $\mathrm{SU}(2)$ coupling. Second, $m_{\phi}{ }^{2}$ is not special at the unification scale, but runs to be negative by the electroweak scale, driven negative by the large top quark Yukawa coupling. Thus the "Mexican hat" potential with a minimum away from $\phi=0$ is derived rather than assumed. As is normal for progress in physics, this explanation is not from first principles, but it is an explanation in terms of the next deeper effective theory, and depends on the supersymmetry breaking leading to soft masses of order the weak scale. That is not circular because the crucial issue is how the $\mathrm{SU}(2) \times \mathrm{U}(1)$ symmetry is broken.

- Supersymmetry can stabilize the hierarchy between the weak scale and the unification scale, and mitigate the naturalness problem, providing a small ratio of the weak scale to the unification scale, because of log running.

- Supersymmetry allows the gauge couplings to unify.

- Supersymmetry can provide a cold dark matter candidate.
- Supersymmetry can explain the baryon asymmetry of the universe.

- Supersymmetry has made several correct predictions:

(1) Supersymmetry predicted in the early $1980 \mathrm{~s}$ that the top quark would be heavy, because that was a necessary condition for the validity of the EWSB explanation.

(2) Supersymmetry correctly predicted the present experimental value of $\sin ^{2} \theta_{W}$ before it was measured.

(3) Supersymmetry requires a light Higgs boson to exist, and the precision measurements lead to $M_{h} \lesssim 200 \mathrm{GeV}$.

(4) When LEP began to run in 1989 it was possible to say that either LEP would discover light superpartners if they were very light, or because all supersymmetry effects at LEP are loop effects, and supersymmetry effects decouple as superpartners get heavier, there would be no significant deviations from the SM discovered at LEP. That is, it is only possible to have loop effects large enough to measure at LEP + SLC if superpartners are light enough to directly observe. In non-supersymmetric approaches it was natural to expect effects at LEP.

It is remarkable that supersymmetry was not invented to explain any of the above physics. Supersymmetry was written as an interesting and beautiful property of theories, studied for its own sake, in the early 1970s. Only after several years of improving the understanding of the theory did people begin to realize that supersymmetry solved the above problems, one by one. It is also remarkable that all of the above successes can be achieved simultaneously, with one consistent form of the theory and its parameters. Finally, it is also noteworthy that low energy softly broken supersymmetry has no known incorrect predictions; it is not so easy to write a theory that explains and predicts some phenomena and has 
no conflict with other phenomena.

Some physicists argue that gauge coupling unification is the most important success of supersymmetry, and it is indeed a major result. But the issue of how to break the electroweak symmetry is the more fundamental problem. Any solution of that problem must simultaneously accommodate both the boson and fermion masses. Explaining the mechanism of EWSB is the deepest reason why we should expect low energy supersymmetry in nature. No other approach should be taken to be of comparable interest to supersymmetry unless it can provide an appropriate explanation of EWSB.

It should already be clear that our framework for these lectures is the traditional one with the Planck scale above $10^{18} \mathrm{GeV}$, and gauge coupling unification somewhat above $10^{16} \mathrm{GeV}$. We are convinced that a consistent quantum theory of gravity and the SM forces requires extra dimensions in some sense, but not that any of them are larger than the (inverse) unification scale. We expect the extra dimensions to be small because the traditional picture based on having a primary theory at the traditional Planck scale, with a hierarchy protected by supersymmetry, provides beautiful, understandable explanations (such as explaining EW symmetry breaking) and successful predictions, while alternative approaches do not. Alternative approaches should be explored, but this approach should remain the default.

String theory can provide a consistent quantum theory of gravity. In addition it can incorporate the known forces and fundamental particles, providing a corresponding theoretical framework. But, string theory will be formulated with extra space-time dimensions. It must be compactified to $4 \mathrm{D}$, and supersymmetry must be broken, to give an effective theory at the unification scale. It is that effective theory that is the focus of these lectures.

The region between the weak or collider scale, and the unification scale, need not be empty indeed, we expect a variety of states in that re- gion, including right-handed neutrinos involved in generating neutrino masses, possible axions, possible vector or SU(5) multiplets, and more. All that is assumed is that the theory remains perturbative in the region from a $\mathrm{TeV}$ or so to the unification scale. There is strong evidence for this assumption - both the unification of the gauge couplings and the explanation of EWSB independently imply that the theory is indeed perturbative in this region.

With this worldview we can divide the physics opportunities into three categories. The foundation is experiments, of course, experiments at colliders such as LEP and the Tevatron and LHC, and b-factories, and rare decays, and proton decay, and electric dipole moment measurements, and cold dark matter detectors. We will discuss all of these in the following. At the other end is the effort to construct a consistent string theory which describes our world, which includes finding out how it is compactified, and how supersymmetry is broken. We will see that there is a mismatch between these two efforts. String theorists and experimenters cannot communicate with each other, but they can communicate through the third category, supersymmetry theorists. The stheorists are needed for two reasons. Experimenters will measure, once superpartners and Higgs bosons are directly observed, some kinematical masses of superpartner mass eigenstates, and some cross sections times branching ratios. Essentially none of the quantities that experimenters measure can emerge from a string theory (as we will see more clearly below). The stheorists will first help convert the observables into the parameters of the supersymmetry Lagrangian, and then will help convert that Lagrangian from its value at the weak scale to its value at the unification scale. That can in turn be compared with the predictions of a compactified string theory with broken supersymmetry. Of course, a given person can contribute in more than one category - for example, experimenters can translate the observables into the Lagrangian parameters, perhaps string theorists can help formulate how to relate the weak scale Lagrangian to the unification scale one, and stheorists can 
help formulate string based models. In practice, however, I will argue that it is highly probable that the historical path will be followed. String theory in 10D has no known testable predictions or explanations of particle physics phenomena in the $4 \mathrm{D}$ world. Probably figuring out how to compactify and how to break supersymmetry is too difficult for anyone to do purely from first principles. But even more, suppose someone had figured it out. How would they convince themselves or anyone else they had done so? Many compactifications or brane worlds have three families, so that is not a unique clue. Most approaches to solving the cosmological constant problem are generic. Calculating the small Yukawa couplings is likely to depend on many difficult details of the theory, as is calculating the mass of the LSP or $\tan \beta$. I am not arguing that string theory is not testable physics, or that we will not be able to make great progress toward becoming confident that string theory is the right approach to discovering the primary theory, but that the crucial steps of learning why 3 space dimensions are large and how supersymmetry is broken will almost certainly only come after there is data on superpartners to point the way. In fact, the main result that will emerge from any tentative compactification and supersymmetry breaking is the soft-breaking supersymmetry Lagrangian, $\mathcal{L}_{\text {soft }}$, with its many parameters. But until $\mathcal{L}_{\text {soft }}$ is measured it will not be possible to recognize its correctness. I expect it will be the other way around. Once $\mathcal{L}_{\text {soft }}$ is measured and translated to the unification scale (a significant challenge), its patterns will lead string theorists to recognize how to compactify and how to break supersymmetry and how to find the correct vacuum. The data and the stheorists will be essential to make progress. At the same time, it will be necessary to have understood and developed string theory itself to have the technology to join onto the data and to recognize the significance of the patterns that emerge from the data. In the following we will look briefly at some of the kinds of patterns and what they might imply. Thus these lectures focus on $\mathcal{L}_{\text {soft }}$.

\section{THE SUPERSYMMETRY SOFT- BREAKING LAGRANGIAN, $\mathcal{L}_{\text {soft }}$ - LARGE PHASES?}

We know that supersymmetry is a broken symmetry, but the physics of supersymmetry breaking is not yet understood. That's not surprising - the symmetry breaking was the last thing understood for the Standard Model (SM) too (assuming we do indeed understand it). For the SM the symmetry breaking originates in a different sector, with the soft-breaking supersymmetry terms that make up the SM effective scalar potential. The symmetry breaking is transmitted to the SM bosons and fermions by interactions with the Higgs field. There were several ways the EW symmetry could be broken — we will not know for sure which is nature's choice until a light Higgs boson is directly observed. In the supersymmetry case there are also several ways the supersymmetry could be broken — we do not know which, if any, is correct, and we do not yet know how the breaking is transmitted to the superpartners. All of this information, both the breaking and the transmission, is encoded in $\mathcal{L}_{\text {soft }}$.

Fortunately, the theory is powerful enough that it is possible to write the "most" general effective Lagrangian [i, [i, even though we do not know the mechanism of supersymmetry breaking. This Lagrangian is defined to include all allowed terms that do not introduce quadratic divergences in the theory, which include all allowed terms of dimension two and three. It is gauge invariant and Lorentz invariant. The Lagrangian depends of course on what the gauge group and particle content are assumed to be. We will study the case of the general supersymmetric Standard Model (SSM), where we assume the theory contains the $\mathrm{SM}$ particles and has the $\mathrm{SM} \mathrm{SU}(3) \times \mathrm{SU}(2) \times \mathrm{U}(1)$ gauge group, has two Higgs doublet fields as is necessary in supersymmetry to give mass to both up and down type quarks, and has a conserved R-parity. "Most" is in quotes above for two reasons. First, the correct theory could be larger than the SSM, and second, some kinds of terms 
are not normally included since they are usually very small in models [2i]. If we wanted to extend the theory, for example to add an extra singlet scalar or an additional U(1) symmetry, we could add the associated terms. Similarly, just as it is necessary to add new fields such as righthanded neutrinos to the SM to incorporate neutrino masses in the SM, we would have to add such fields and their superpartners and the associated terms in $\mathcal{L}_{\text {soft }}$ if we wanted to include neutrino masses in our theory. We will not do that in these lectures.

The situation is analogous to that in the SM with the quark masses and the CKM matrix which contains flavor mixing angles and a phase. In the supersymmetric case there are parameters that are masses, flavor rotation angles, and phases. Just as for the CKM matrix, all these parameters have to be measured, unless a compelling theory determines them eventually. Before the top quark mass was known, we could assume a value for the top quark mass and then calculate its production cross section, its decay branching ratios and signatures, and all aspects of its behavior. Since all other needed SM parameters were measured only the top mass was unknown; if some other SM parameters had not yet been measured we would have had to choose various values for them too. The situation for superpartners is similar - for any given set of masses and flavor mixing angles and phases we can calculate the observable aspects of superpartner behavior. We can study any tentative supersymmetry signal and decide if it is consistent with the theory, we can make predictions and plan for future facilities, and much more. $\mathcal{L}_{\text {soft }}$ will contain at least 105 new parameters, depending on what we include. That might seem like a lot, but almost all have clear physical interpretations. Once there is data most will be measured, and their patterns will quickly reduce the number. The historical situation with the SM was similar. Once we knew the effective Lagrangian was $V-A$ many parameters disappeared, and such a structure led to recognizing it was a gauge theory which reduced the number more. Probably the situation will be similar for supersymmetry.
[Counting parameters depends on assumptions. One reasonable way to count the SM parameters for comparison with supersymmetry is to assume that all the particles are known, but not their masses or interactions. Then the $\mathrm{W}$ and $\mathrm{Z}$ vertices can each have a space-time tensor character of scalar, vector, etc (, V, T, A, P), and each can be complex (so multiply by 2 ). Conserving electric charge, the $\mathrm{Z}$ can have 12 different flavornon-changing vertices for the 12 quarks and leptons (e, $\left.\mu, \tau, \nu_{e}, \nu_{\mu}, \nu_{\tau}, u, c, t, d, s, b\right)$, plus 12 additional flavor-changing vertices $(e \mu, e \tau, \mu \tau$, etc.). This gives 240 parameters $(12 \times 5 \times 2 \times 2)$. Similar counting for the $\mathrm{W}$ gives 180 . There are 12 masses. Self-couplings of $\mathrm{W}$ and $\mathrm{Z}$ allowing $\mathrm{CP}$ violation give 10 . The total here is 442 parameters.]

We can write the soft Lagrangian as

$$
\begin{aligned}
& -\mathcal{L}_{\text {soft }}=\frac{1}{2}\left(M_{3} \tilde{g} \tilde{g}+M_{2} \tilde{W} \tilde{W}+M_{1} \tilde{B} \tilde{B}+\text { h.c. }\right) \\
& +\tilde{Q}^{\dagger} M_{\tilde{Q}}^{2} \tilde{Q}+\tilde{U}^{c \dagger} M_{\tilde{U}}^{2} \tilde{U}^{c}+\tilde{D}^{c \dagger} M_{\tilde{D}}^{2} \tilde{D}^{c}+\tilde{L}^{\dagger} M_{\tilde{L}}^{2} \tilde{L}+\tilde{E}^{c \dagger} \\
& M_{\tilde{E}}^{2} \tilde{E}^{c}+\tilde{+} U^{c} \tilde{A}_{U} \tilde{Q} H_{U}+\tilde{D}^{c} \tilde{A}_{D} \tilde{Q} H_{D}+\tilde{E}^{c} \tilde{A}_{E} \tilde{L} H_{D} \\
& + \text { h.c. }+m_{H_{U}}^{2} H_{U}^{*} H_{U}+m_{H_{D}}^{2} H_{D}^{*} H_{D}+\left(b H_{U} H_{D}+h . ф 2.1\right)
\end{aligned}
$$

Supersymmetry is broken because these terms contribute explicitly to masses and interactions of (say) winos or squarks but not to their superpartners. The underlying supersymmetry breaking is assumed to be spontaneous, but its effects are explicit in this effective Lagrangian. Similarly, how supersymmetry breaking is transmitted to the superpartners and their interactions is encoded in the parameters of $\mathcal{L}_{\text {soft }}$. All the quantities in $\mathcal{L}_{\text {soft }}$ are scale dependent and satisfy known renormalization group equations. $\mathcal{L}_{\text {soft }}$ has the same form at any scale. All of the coefficients in $\mathcal{L}_{\text {soft }}$ - the "soft" parameters - can be complex, as long as the Lagrangian is Hermitian.

The gaugino masses $M_{i}$ can be written as $M_{3}=$ $\left|M_{3}\right| e^{i \phi_{3}}$, etc. From now on in these lectures we will for simplicity write $M_{3}$ for the magnitude and assume the phase is explicitly present. 
The squark and slepton mass matrices of Eq.(2.1) are Hermitian matrices, so the diagonal elements are real, while the off-diagonal elements can be complex. The absence of flavor changing neutral decays of quarks and leptons puts strong constraints on these off-diagonal elements [3i]. The trilinear or triscalar couplings $\tilde{A}_{U, D, E}$ are general $3 \times 3$ complex matrices in flavor space.

Symmetries of the theory allow some of the parameters to be absorbed or rotated away with field redefinitions. The net number left $\left[\overline{4} \overline{1}_{i}\right]$ is 103. If the gravitino is the lightest superpartner (LSP) then its mass and phase have to be included. Finally, although there is no bare $\mu$ term in the superpotential in a string-based approach, because the terms present are associated with massless strings, it is both necessary phenomenologically and expected theoretically that an effective $\mu$ term will be generated, with the magnitude of $\mu$ of a size similar to the other soft terms. If there were no effective $\mu$ term, the Higgs sector would have a massless pseudoscalar that does not exist. In general there will also be a phase $\phi_{\mu}$.

Finally, then, we will examine the behavior of phenomena based on supersymmetry physics that results from a superpotential with Yukawa coupling and an effective $\mu$ term,

$W=y_{U} Q U^{c} H_{U}+y_{D} Q D^{c} H_{D}+y_{E} L E^{c} H_{D}+\mu_{e f f}$

plus a soft Lagrangian $\mathcal{L}_{\text {soft }}$ (from now on we write $\mu_{\text {eff }}=\mu$ ).

We will focus on the phases of the soft parameters in these lectures, for several reasons. They are the least studied aspects of the soft parameters. (The off-diagonal flavor structure of the squark and slepton soft masses and of the triscalar terms is also little-studied and quite interesting.) If the phases are large they can have very large effects [5] on a variety of interesting phenomena - they generate $\mathrm{CP}$ violation, they affect the baryon asymmetry of the universe, the relic density and detectability of cold dark matter, rare decays, implications of the Higgs sector, and superpartner masses, cross sections, and branching ratios. The patterns of the phases, and whether they are measured to be large or small, will point to compactifications on different manifolds and to different ways to break supersymmetry. We now understand how to measure most of the phases. In keeping with the view that the phases must first be measured before their implications for compactification and supersymmetry breaking can be understood, we will emphasize in the following how to measure the phases and how they affect observables. For a very large variety of particle physics and cosmology phenomena one can be badly misled if phases are large but are not included in analyses.

\section{HOW DO THE SOFT PARAM- ETERS SHOW UP IN EXPERI- MENTS?}

Let's suppose superpartners and Higgs bosons are found. First let's list the masses of the particles that will eventually be seen. There are 4 neutralino masses, associated with the soft terms from $W^{0}, B^{0}, H_{U}^{0}, H_{D}^{0}$ (or, in the mass eigenstate basis, $\left.\gamma, Z, H_{U}^{0}, H_{D}^{0}\right)$. The neutralino superpartners mix, so the physical neutralino mass eigenstates $\tilde{N}_{1,2,3,4}$ will be the eigenvalues of a mass $H_{a}$ at there are 2 chargino mass eigenstates from the chargino mass matrix. There are 4 Higgs boson masses, for $h^{0}, H^{0}, A^{0}, H^{ \pm}$. There is 1 gluino mass, and 1 gravitino mass. The squark mass matrix for up-type squarks has 6 independent eigenvalues, the superpartners of the left- and right-handed quarks u,c,t: $\tilde{u}_{L}, \tilde{c}_{L}, \tilde{t}_{L}, \tilde{u}_{R}, \tilde{c}_{R}, \tilde{t}_{R}$. Similarly, there are 6 down-type mass eigenstates and 6 charged lepton mass eigenstates. In the SSM there are only the three left-handed neutrinos and their sneutrinos. These add up to 33 physical masses that can be measured if all the states are found in experiments. A 34th mass is the gravitino mass; if the gravitino is not the LSP then it may not be possible to measure its 
mass since it couples too weakly to be produced directly at colliders, and affects only some earlyuniverse cosmology.

Another parameter is $\tan \beta$, the ratio of the vacuum expectation values (vev's) of the two Higgs fields,

$$
\tan \beta=\left\langle H_{U}\right\rangle /\left\langle H_{D}\right\rangle .
$$

$\tan \beta$ is intrinsically a low energy parameter, since the Higgs fields do not have vev's until the RGE running induces them somewhat above the weak scale. We will see that measuring $\tan \beta$ is in general difficult, and cannot be done accurately without a lepton collider with a polarized beam that is above the threshold for several superpartners. When we are trying to deduce the unification scale Lagrangian $\tan \beta$ can be traded for a high scale parameter in the Higgs sector.

43 of the parameters in $\mathcal{L}_{\text {soft }}$ are phases. As we have mentioned, they affect essentially all observables. Knowledge of the phases could provide the essential clues to how to compactify and break supersymmetry.

Phenomenologically, life would be much simpler if the phases were zero, or small. It would be much easier to determine the soft parameters from data, to measure $\tan \beta$, etc. Are there reasons to think the phases might be small? Until recently

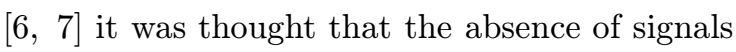
for electric dipole moments (EDMs) of neutrons and electrons implied that some flavor diagonal phases were small [in. These arguments concerning EDM phases would not impact many of the phases even if they did apply. Basically, if all phases and soft parameters were considered as independent and if first-family squarks and sleptons were not heavier than a few hundred $\mathrm{GeV}$, then large phases would imply EDMs larger than the experimental limits. Of course the first family squarks and sleptons could be heavy, suppressing EDM's, but let us assume they are not.

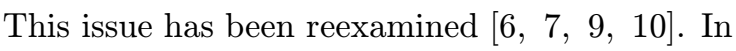

a real theory it would necessarily occur that various soft terms and phases are related, perhaps originating from a few F-term vev's or some other mechanism. Some cancellations are automatic but were not included in the original estimates, such as those between charginos and neutralinos arising from the automatic negative sign in the Lagrangian between the $H_{U}^{+} H_{D}^{-}-H_{U}{ }^{0} H_{D}{ }^{0}$ terms. Others have been shown to arise in models (such as the D-brane model [1] 10 ] presented later in the lectures). Some people have worried that the needed cancellations are a fine-tuning. That is not so - any theory of the soft parameters will give relations among them. What would happen here if such cancellations indeed occurred is somewhat analogous to the GIM mechanism. If such cancellations occur, it will not be because of an obvious symmetry, but rather a symmetry at the string scale that leads to relations among the soft parameters. The bottom line is that the flavor-diagonal phases could well be large and still be consistent with current EDMs. However, if the EDM experiments improve approximately an order of magnitude and still do not see a signal then the phases they constrain are probably small.

At present there are also reasons why it seems that the phases may be large. In general this has to do with how $\mathrm{CP}$ violation effects originate.

A. The baryon asymmetry of the universe cannot be explained in the SM [1] 1, n, so some other significant phases are needed. The role of CP violation in various ways of generating a baryon asymmetry is not yet well enough understood to be sure of the origin of the phases. The supersymmetry soft phases provide the $\mathrm{CP}$ violation if the baryon asymmetry is generated at the electroweak phase transition, and perhaps also if the baryon asymmetry is generated by an AffleckDine mechanism. If the baryon asymmetry is generated at the EW phase transition, the size of the needed phases depends on how much washout occurs, and on theoretical issues that are not yet settled. Large phases may be required. In the case of decay of heavy Majorana neutrinos there is no concrete model that allows one to dis- 
cuss the origin of the $\mathrm{CP}$ violation definitively, and it could be from soft phases or from lepton Yukawas. Explaining the baryon asymmetry is certainly easier with large soft phases, and may require them.

B. Normally it is assumed that the SM CKM phase accounts for $\mathrm{CP}$ violation in the Kaon system, which requires a large CKM phase, and the b-factories will study whether the CKM phase describes $\mathrm{CP}$ violation in the $\mathrm{B}$ system. If the CKM phase turns out to be small [1]2'], supersymmetry soft phases are then clearly needed to describe CP violation in the Kaon and B systems (they can do that, see below). Since the Yukawa couplings enter the superpotential, and are present even if supersymmetry is unbroken, it is easy to imagine that the Yukawa couplings are relatively real so that the CKM phase is zero (it may get small renormalization effects at the weak scale but would still be effectively zero numerically). The soft phases can enter as a result of the supersymmetry breaking, which can be transmitted to the observable sector by complex vev's of F-terms, analogous to complex Higgs vev's, giving complex soft terms but relatively real Yukawas.

C. If the CKM phase is large, more data is needed to see if there is evidence for soft phases in the Kaon and B systems. But if the CKM phase is large and one looks at how the soft phases are calculated from the superpotential (and Kahler potential and gauge kinetic function), it is easy to see how the soft phases could be large [1] $3_{1}^{\prime}$. In general, the Yukawas are functions of moduli that get possibly complex vev's, and soft triscalar terms and gaugino masses are functions of those moduli vev's and also F-term vev's that can be complex. In particular, the triscalar $\tilde{A}$ terms are linear combinations of Yukawas and derivatives of Yukawas, so if the CKM phase is large it is likely the $\tilde{A}$ phases are large (care must be taken here to be precise about whether one is discussing the $\tilde{A}$ of Eq.(3.1) or the coefficients $A_{i j k}$ if one writes $\left.\tilde{A}_{i j k}=A_{i j k} Y_{i j k}\right)$. If the vev's that give rise to the spontaneous supersymmetry breaking are complex, it is very likely some soft phases will be large.

The form for $\mathcal{L}_{\text {soft }}$ is rather general, and allows for some other possibly important effects, such as D-terms (from the breaking of extra U(1) symmetries) that give contributions to squark and slepton masses, or Planck scale operators that lead to contributions to masses when some fields get vev's. Extra U(1)'s or extra scalars can lead to a larger neutralino mass matrix than the $4 \mathrm{x} 4$ one expected here. Terms of the form $\phi^{*} \phi^{2}$ (rather than $\phi^{3}$ ) are generally allowed [2in in gauge theories where the scalars are charged under some gauge group, but no models are yet known where such terms give significant effects. They can be added if necessary.

I have said that it is very unlikely that string theorists could guess the correct compactification and supersymmetry breaking mechanism and convince themselves or anyone else that they had the answer. There is an additional difficulty with a top-down approach. To predict any weak scale observable it is necessary to know several soft parameters, as we will see in detail below. In addition, to run down to the weak scale it is usually necessary to know several more. Further, the $\mu$ parameter is likely to have a different physical origin (e.g. from the Kahler potential as in the Giudice-Masiero mechanism) and it also has to be known. The phases have to be known. Finally, it is necessary to calculate $\tan \beta$ which requires additional soft parameters and their running. Basically, it is necessary to get it all right at once to predict any weak scale observable correctly. Conversely, if any part of the assumed compactification and supersymmetry breaking approach is wrong, predictions for some or all observables are likely to be wrong in practice. This reinforces the view that it is likely that it will be necessary to measure the parameters of $\mathcal{L}_{\text {soft }}$ at the weak scale in order to formulate string theory in $4 \mathrm{D}$ with broken supersymmetry. 


\section{MEASURING THE SOFT PAR- AMETERS}

Consequently, let us turn to how to connect the soft parameters with observables. The essential point is that experimenters measure kinematical masses, cross sections times branching ratios, electric dipole moments, etc. We have to examine how those are expressed in terms of soft parameters. We will see that at most two of the soft parameters can be directly measured, the gluino and gravitino masses. The gravitino mass can probably only be measured if it is the LSP, and then only very approximately. The soft parameter $M_{3}$ can be deduced from the gluino mass to about $20 \%$ accuracy from theoretical uncertainties [1 14 is associated with large loop corrections depending on squark masses (not counting experimental uncertainties).

\section{THE CHARGINO SECTOR}

An important case, and the simplest example, is the chargino sector. The superpartners of $W^{ \pm}$ and of the charged Higgs bosons $H^{ \pm}$are both spin-1/2 fermions and they mix once the electroweak symmetry is broken, i.e. once the neutral Higgs field get vev's. There is a $W \widetilde{W}$ mass term $M_{2} e^{i \phi_{2}}$, a Higgsino mass term $\mu e^{i \phi_{\mu}}$, and a mixing term, so the chargino mass matrix is

$$
M_{\tilde{C}}=\left(\begin{array}{cc}
M_{2} e^{i \phi_{2}} & \sqrt{2} M_{W} \sin \beta \\
\sqrt{2} M_{W} \cos \beta & \mu e^{i \phi_{\mu}}
\end{array}\right) .
$$

The eigenvalues of this matrix (since it is not symmetric one usually diagonalizes $M_{\tilde{c}}^{\dagger} M_{\tilde{c}}$ ) are the physical mass eigenstates, $M_{\tilde{C}_{1}}$ and $M_{\tilde{C}_{2}}$. The formulas are a little simpler if we write them in terms of the trace (sum of eigenvalues) and determinant (product of eigenvalues),

$\left.\operatorname{Tr} M_{\tilde{C}}^{\dagger} M_{\tilde{C}}=M_{\tilde{C}_{1}}^{2}+M_{\tilde{C}_{2}}^{2}=M_{2}^{2}+\mu^{2}+2 M_{W^{2}}^{2}, 2\right)$
$\operatorname{Det}_{\tilde{C}}^{\dagger} M_{\tilde{C}}=M_{\tilde{C}_{1}}^{2} M_{\tilde{C}_{2}}^{2}=M_{2}^{2} \mu^{2}+2 M_{W}^{4} \sin ^{2} 2 \beta$

$$
-2 M_{W}^{2} M_{2} \mu \sin 2 \beta \cos \left(\phi_{2}+\phi_{\mu}\right) .
$$

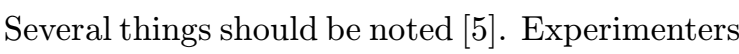
will measure the physical masses $M_{\tilde{C}_{1}}$ and $M_{\tilde{C}_{2}}$. But what we need to know for the Lagrangian are $M_{2}, \mu$, and the phases. We also need to measure $\tan \beta$. The phases enter here only in the combination $\phi_{2}+\phi_{\mu}$. This is a physical phase that cannot be rotated away, a true observable, as much as the CKM phase of the quark mixing matrix. Such "reparameterization invariant" combinations of phases enter all observables. In general we think of phases as leading to CP-violating phenomena, and they do, but note that here the (non-CP-violating) masses also depend strongly on the phases.

After diagonalizing this matrix, the symmetry eigenstates can be expressed in terms of the mass eigenstates, which will be linear combinations whose coefficients are the elements of the eigenvectors of the diagonalizing matrix. These coefficients also depend on $\tan \beta$ and the phases, and enter the Feynman rules for producing the mass eigenstates. Thus the cross sections and decay branching ratios (BR) also depend on the phases and $\tan \beta$.

To measure any of the parameters, such as $\tan \beta$, it is necessary to invert the equations and measure all of them. Since there are four parameters here one has to have at least four observables. In practice more observables will be necessary since there will be quadratic and trigonometric ambiguities, and since experimental errors will lead to overlapping solutions. Thus we learn the important result [i5i] that from the masses alone we cannot measure $\tan \beta$. 
It is worth elaborating on this point, because many papers and phenomenological analyses have claimed that $\tan \beta$ can be measured in various sectors. Whenever that is done (except at a lepton collider with polarized beams or by combining a variety of Higgs sector data - see below) the analysis has actually assumed various soft terms are zero or equal, to reduce the number of parameters. While such assumptions may (or may not) be good guesses, once there is data it is necessary to measure such parameters without assumptions.

The next thing to try is to add the (presumed) cross section data. The dominant processes are s-channel $\mathrm{Z}$ and $\gamma$, and sneutrino exchange, as shown (they are shown for $e^{+}, e^{-}$beams - similar diagrams can be drawn for hadron colliders):

The couplings to $\mathrm{Z}$ and $\gamma$ are determined by the diagonalized mass matrix, but now the sneutrino masses and couplings enter. If we make no assumptions there are three sneutrino masses, each with its own coupling, so 6 new parameters; actually, the sum of squares of the couplings is determined by supersymmetry to be equal to the $\nu \mathrm{eW}$ coupling, so there are only 5 new parameters. If we don't consider chargino decays, there are three cross sections, $\tilde{C}_{1} \tilde{C}_{1}, \tilde{C}_{2} \tilde{C}_{2}, \tilde{C}_{1} \tilde{C}_{2}$. In principle, one can imagine measuring differential cross sections, obtaining several angular bins. In practice, with limited statistics and backgrounds, usually even at an electron collider one only measures forward-backward asymmetries $\mathcal{A}$. At a hadron collider it would be very hard to measure even the asymmetries (because of difficulties in reconstructing the superpartners from their decay products, and because of large backgrounds), and before they were included in the counting a careful simulation would have to be done. Thus, if the produced charginos can be reconstructed, it may be possible to measure $\tan \beta$ at an electron collider [1.5], but probably still not at a hadron collider. However, it needs to be shown that the produced charginos can be reconstructed even at a lepton collider.

Further, the charginos of course decay. There are a number of possible channels, some of which are shown:

These introduce new parameters, slepton and squark masses and couplings, and the LSP mass and couplings, even assuming the decay to the LSP dominates over decay cascades through other neutralinos. Unless one decay dominates, too many parameters may enter to measure $\tan \beta$ from these channels even at a higher energy lepton collider.

If the decay via an intermediate $W$ dominates, some final polarization can be obtained, but if sleptons and squarks are light and contribute to the decays then no polarization information is transmitted to the final state because they are spinless. Their chirality can still enter since the wino component of charginos couples to LH sferm ions, while the Higgsino component couples to $\mathrm{RH}$ sfer mions.

In general then it is not possible to measure $\tan \beta$ or the soft phases or other soft parameters from chargino channels alone, though if squarks and sleptons are heavy or if charginos can be reconstructed experimentally it may be possible [1] If one assumes values for phases or assumes relations for parameters the results for $\tan \beta$ and other parameters are not true measurements and may not correspond to the actual values. However, if one restricts the assumptions to highly plausible ones it is worthwhile to make them and learn as much as possible. Assumptions that are highly plausible include assuming that the three sneutrinos are approximately degenerate, that $\tilde{e}_{L}, \tilde{\mu}_{L}, \tilde{\tau}_{L}$ are approximately degenerate and similarly $\tilde{e}_{R}, \tilde{\mu}_{R}, \tilde{\tau}_{R}$ are approximately degenerate, with similar assumptions for the squarks. Also, for collider physics the first two families can be taken to have small L-R mixing, since L$\mathrm{R}$ mixing is expected to be proportional to the mass of the associated fermions and thus small. Under these assumptions it will definitely be possible to measure $\tan \beta$ and the soft phases at lepton colliders that can produce some of the superpartners, when the extra observables from beam polarization and a second energy are included, even if the collider does not have enough energy 
to produce many superpartners (see Section 14). With such assumptions it may even be possible to measure $\tan \beta$ and some phases at hadron colliders. Some of the assumptions can be checked independently. I will call these approximate approaches "reasonable approximate models".

We have only looked at the chargino channels to have a simple example, but of course all the accessible superpartners will be produced at any collider, leading to more parameters and more observables. We will see below how the counting works for a few kinds of processes, and how channels can be combined. Only with good simulations (or of course real data) can one be confident about counting observables. My estimates are that with hadron colliders true measurements of $\tan \beta$ and soft phases and other soft parameters are not possible, but they may be possible for reasonable approximate models depending on the actual values of the parameters. For lepton colliders with a polarized beam, above the threshold for some superpartners, the parameters of $\mathcal{L}_{\text {soft }}$ can be measured, as discussed further below.

\section{NEUTRALINOS}

If charginos are produced, neutralinos will be produced too. So we get more observables (masses, cross sections, asymmetries). There are more parameters too, but not as many new parameters as new observables. The symmetric neutralino mass matrix is
We saw that the chargino sector depended on a single physical phase, reparameterization invariant, $\phi_{2}+\phi_{\mu}$. Similarly, there are two physical phases, that cannot be rotated away, in the neutralino matrix. One can see this various ways - one is by simply calculating observables. A somewhat easier one is by redefining the basis by

$$
\left(\begin{array}{llll}
e^{-i \phi_{\mu} / 2} & & & \\
& e^{-i \phi_{\mu} / 2} & & \\
& & e^{-i \phi_{2} / 2} & \\
& & & e^{-i \phi_{2} / 2}
\end{array}\right)
$$

in which case the resulting matrix depends explicitly only the physical phases.

So there is one new soft mass, $M_{1}$, and one new physical phase, $\phi_{1}+\phi_{\mu}$. In principle the masses of the four mass eigenstates can be measured, and the cross sections $\tilde{N}_{1}+\tilde{N}_{1}, \tilde{N}_{1}+\tilde{N}_{2}, \tilde{N}_{2}+\tilde{N}_{2}$, etc. and associated asymmetries. The number of new observables is different at different colliders. Below we will also discuss the number of parameters and observables from the Higgs sector explicitly. It is extremely important for detector groups at various colliders to count the number of observables they can expect to measure. This has to be done using models, of course, so it is very important that the models be ones that can describe the electroweak symmetry breaking without excessive fine tuning. Of course, the models should also be consistent with LEP data.

\footnotetext{
7. SOME EFFECTS OF PHASES FOR THE HIGGS SECTOR - TRUE

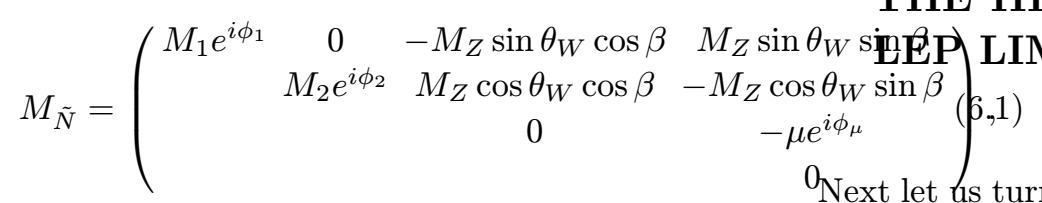

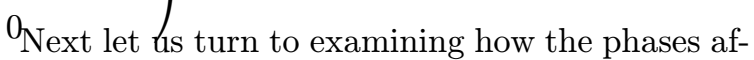

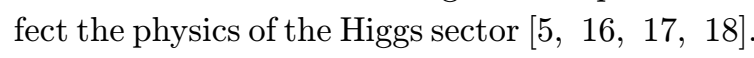
It is important to realize that the phases that enin the symmetry basis $\left(\tilde{B}, \tilde{W}, \tilde{H}_{U}, \tilde{H}_{D}\right)$. Even ter the Higgs sector are not new ones, they are some of the same ones that are already in $\mathcal{L}_{\text {soft }}$. when the elements are complex it can be diagonalized by a single unitary matrix.
} 
the quantities that affect the Higgs physics can be chosen to be real. The phase effects enter at one loop order, because the stop loops are a large

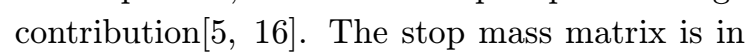
general complex, just as the chargino mass matrix is complex, and so the phases enter into the scalar effective potential.

One can write

$$
\begin{aligned}
H_{D} & =\frac{1}{\sqrt{2}}\left(\begin{array}{c}
\mathrm{v}_{D}+h_{1}+i a_{1} \\
h_{1}^{-}
\end{array}\right), \\
H_{U} & =\frac{e^{i \vartheta}}{\sqrt{2}}\left(\begin{array}{c}
\mathrm{v}_{U}+h_{2}+i a_{2} \\
h_{2}^{+}
\end{array}\right),
\end{aligned}
$$

with the vev's taken to be real, and $\tan \beta=\mathrm{v}_{U} / \mathrm{v}_{D}$. The phase $\vartheta$ allows a relative phase between the two vev's at the minimum of the Higgs potential. The stop mass matrix has off diagonal L-R elements

$$
y_{t}\left(A_{t} H_{U}-\mu^{*} H_{D}^{*}\right)
$$

so the phases of the triscalar coupling $A_{t}$ and of $\mu$ and the relative phase $\vartheta$ enter the stop mass eigenvalues. The scalar potential contains a term

$$
\sum m_{\tilde{t}_{i}}^{4} \ln m_{\tilde{t}_{i}}^{2}
$$

and the phases $\phi_{A_{t}}, \phi_{\mu}$ enter through the stop mass eigenvalues $m_{\tilde{t}_{i}}$. The scalar potential $V$ is then minimized by setting $\partial V / \partial h_{1}, \partial V / \partial h_{2}, \partial V /$ $\partial a_{1}, \partial V / \partial a_{2}=0$. Two of the resulting four equations are not independent, so three conditions remain.

The Higgs sector then has 12 parameters, $\mathrm{v}_{U}, \mathrm{v}_{D}$, $\phi_{A_{t}}, \phi_{\mu}, \vartheta, A_{t}, \mu$, the parameters $m_{\tilde{Q}}^{2}, m_{\tilde{U}}^{2}, b, m_{H_{U}}^{2}, m_{H}$ from $\mathcal{L}_{\text {soft }}$, and the renormalization scale $Q$ since the parameters run. Three can be eliminated by the three equations from minimizing $V$. The scale $Q$ is chosen to minimize higher order corrections. The conditions that guarantee EWSB occurs let us replace $\mathrm{v}_{U}, \mathrm{v}_{D}$ by $M_{Z}$ and $\tan \beta$ as usual. Thus there are 7 physical parameters left, including $\tan \beta$ and one physical (reparameterizationinvari ant) phase $\phi_{A_{t}}+\phi_{\mu}$. This number cannot be reduced without new theoretical or experimental information. Any description of the Higgs sector based on fewer than 7 parameters has made arbitrary guesses for some of these parameters, and is likely to be wrong. If $\tan \beta$ is large, then sbottom loops can also enter $V$ and additional parameters are present.

If the phase is non-zero it is not possible to separate the "pseudoscalar" $A=\sin \beta a_{1}+\cos \beta a_{2}$ from $h, H$ so it is necessary to diagonalize a $3 \times 3$ mass matrix. We name the three mass eigenstates $H^{i}$; in the limit of no CP-violating phase $H^{1} \rightarrow h, H^{2} \rightarrow A, H^{3} \rightarrow H$. Then, of course, all three mass eigenstates can decay into any given final state or be produced in any channel, so there could be three mass peaks present in a channel such as $Z+$ Higgs (wouldn't that be nice). All production rates and branching ratios depend on the phase, and can change significantly.

It is particularly interesting to ask two questions [1] 1 in. (1) Suppose there is no Higgs boson found at LEP. Then there is an experimental limit on $\sigma\left(H^{1}\right) \times B R\left(H^{1} \rightarrow b \bar{b}\right)$. What are the lower limits on $m_{H^{1}}$ and on $\tan \beta$ in the full 7 parameter theory compared to the SSM with the phase set to 0 or $\pi$ ? This is answered in Figure 7.1. We see that the mass of the lightest Higgs boson is allowed to be significantly lighter if the phase is present. To summarize, the lower limit for the SSM without phases is about $10 \%$ below the SM limit, and the lower limit with the phase present is an additional $10 \%$ lower (about $85 \mathrm{GeV}$ ). Similarly, $\tan \beta$ can take on lower values if the phase is non-zero, probably values down to about 1.5 or even below, so very little of the perturbatively allowed range of $\tan \beta$ is excluded, contrary to what is often asserted by LEP experimental groups or theorists $H_{\text {国nalyzing the data. }}$ 
(2) Suppose a Higgs boson is found. Then $m_{H^{1}}$ and its $\sigma \times B R$ are measured. What region of the full 7 parameter space is allowed? Again the answer is different for the case with phase set to $0, \pi$ and the case with general phase allowed, as shown in Figure 7.2. Thus once there is a discovery it will be seriously misleading to not include the phase in the analysis. The quantity $2 m_{3}^{2} \sin 2 \beta$ plotted on the horizontal axis is what would be $m_{A}^{2}$ if the phase were $0, \pi$.

In both cases the results shown here are simply meant to illustrate the effect of the phase. The full range of the other parameters is not covered, and experimental aspects are not included except for crude estimates of efficiencies. The proper analyses need to be done by experimenters. If the heavier Higgs bosons are heavy, so that they decouple, the effect decreases for the lower limit on the mass of the lightest eigenstate, but there is still a significant effect on the lower limit on $\tan \beta$.

With 7 parameters we would need at least 7 observables if we were to determine (say) $\tan \beta$ from the Higgs sector alone. The possible observables are the 3 neutral scalar mass eigenstates, the charged Higgs mass, the three $\sigma \times B R$ for $Z+$ Higgs and three $\sigma \times B R$ for channels $H^{i}+H^{j}$, and the two stop mass eigenstate masses. Probably in addition one can add the ratio $r=\sigma\left(g g \rightarrow H^{2} \rightarrow\right.$ $b \bar{b}) / \sigma\left(g g \rightarrow H^{1} \rightarrow b \bar{b}\right)$. So eventually it will be possible to measure $\tan \beta$ and the phase $\phi_{A_{t}}+\phi_{\mu}$ in the Higgs sector and compare with the $\tan \beta$ measured in the gaugino sector. LHC data may allow one to carry out such an analysis.

\section{THE GLUINO PHASE}

The phase of the gluino or SU(3) gaugino nicely illustrates some of the subtleties of including and measuring the phases $\left[{ }_{1}^{1} \overline{9}_{1}^{\prime}\right]$. People have wondered if it could be rotated away, or if it was not measurable. More precisely, only certain combinations of phases are invariant under phase redefinitions, as we saw above and as we will see further below. The gluino phase $\phi_{3}$ is not itself a physical observable phase. In these lectures we will not discuss this issue in detail, but simply find observable sets of phases in pratical situations. The general analysis is understood, and can be found in refrences [20, measure $\phi_{3}$ (relative to other phases) below. In the two component notation that is often used in the supersymmetry formalism, the gluino part of the Lagrangian is

$$
\mathcal{L}=-\frac{1}{2}\left(M_{3} e^{i \phi_{3}} \lambda \lambda+M_{3} e^{-i \phi_{3}} \bar{\lambda} \bar{\lambda}\right)
$$

where $\lambda$ is the gluino field. It is inconvenient to have complex masses in the Feynman rules, so we redefine the field,

$$
\psi=G \lambda, \bar{\psi}=G^{*} \bar{\lambda}
$$

where $G=e^{i \phi_{3} / 2}$. Then for any flavor the Feynman rules introduce factors of $G$ or $G^{*}$ at the vertices,

not including the color factors.

Now consider gluino production $q+\bar{q} \rightarrow \tilde{g}+\tilde{g}$. Factors of $G$ and $G^{*}$ enter so that there is no dependence on the phase from these two diagrams.

If we consider $q+g \rightarrow \tilde{q}+\tilde{g}$, production of $\tilde{q}_{L}$ leads to an overall factor of $G^{*}$, while production of $\tilde{q}_{R}$ gives an overall $G$. The phase is then observable if $L-R$ mixing occurs, but $L-R$ mixing is expected to be very small for the first two families (because it is proportional to the off-diagonal element of the sfermion mass matrix, which is usually taken to be proportional to the fermion mass), and those constitute the beams we have to use. Thus gluino production above does not seem to depend on the phase.

But gluinos have to decay, and then the phases 
enter. For simplicity, imagine the gluino decay is via a squark to $q \bar{q} \tilde{\gamma}$, as shown for $\tilde{q}_{L}$,

Then a factor $e^{i \phi_{3} / 2}$ enters at the gluino vertex, and a factor $e^{-i \phi_{1} / 2}$ at the photino vertex. The resulting differential cross section is

$$
\frac{d \sigma}{d x}=32 g_{3}^{2}\left(e e_{q}\right)^{2}\left(\frac{1}{m_{\tilde{q}_{L}}^{4}}+\frac{1}{m_{\tilde{q}_{R}}^{4}}\right) m_{\tilde{g}}^{4} x \sqrt{x^{2}-y^{2}}
$$$$
\left(x-\frac{4}{3} x^{2}-\frac{2}{3} y^{2}+x y^{2}+y\left(1-2 x+y^{2}\right) \cos \left(\phi_{3}-\right.\right.
$$

where $x=E_{\tilde{\gamma}} / m_{\tilde{g}}, y=m_{\tilde{\gamma}} / m_{\tilde{g}}$. Note that what enters is the physical, reparameterization invariant phase $\phi_{3}-\phi_{1}$. Reference [1 $\left.19_{1}^{n}\right]$ has studied how various distributions depend on this phase and on $\tan \beta$ and the soft masses so measurements can be made at the Tevatron and LHC.

\section{CP VIOLATION}

We have looked at some examples of how the phases affect masses, cross sections, and branching ratios (their effects in the Higgs sector involve $\mathrm{CP}$ violating aspects, but somewhat indirectly). Now let us turn to the unique effects they have on $\mathrm{CP}$ violation. We will see that soft supersymmetry phases could explain all the $\mathrm{CP}$ violation in the Kaon and B systems.

How do we expect CP violation to arise in a fundamental way? In string theory, Strominger and

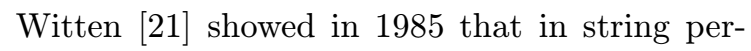
turbation theory CP was a good symmetry, that could be spontaneously broken by moduli vev's. Then in 1992 Dine, Leigh, and MacIntire pointed out $\overline{2} \overline{2}$ that $4 \mathrm{D}$ CP transformations are discrete gauge transformations inherited from 10D string theory, so even non-perturbatively there will be no explicit breaking of CP. Thus CP violation can arise from spontaneous breaking that leads to (a) complex Yukawa couplings that in turn lead to a CKM phase, $\delta_{C K M}$, and (b) the phases of $\mathcal{L}_{\text {soft }}$.

Since the CKM phase cannot explain the baryon asymmetry there must be some other phases that are important, and the soft phases are a leading candidate. Therefore, it is very interesting to examine the possibility that the CKM phase is actually very small, and all CP violation arises from the soft phases. Since the CKM phase arises from the supersymmetry-conserving superpotential if the Yukawa couplings have a relative phase, while the soft phases arise from the supersymmetry$\phi$ (\$) 3r)eaking, it is easy to imagine such a world, and we will examine it phenomenologically. The possibility that all CP violation arose from soft supersymmetry phases [12] was first suggested by Frere and Gavela in 1983, and reexamined by Frere and Abel in 1997.

There are currently six pieces of experimental information on $\mathrm{CP}$ violation. We can compare the SM and a possible version of supersymmetry with large flavor-independent phases and significant off-diagonal triscalar couplings:

\begin{tabular}{ccc}
\hline DATA & SM & ASUPERSYMMETRY \\
\hline$\varepsilon_{K}$ & OK & OK $\left.\delta_{C K M}=0\right)$ \\
$\left(\varepsilon^{\prime} / \varepsilon\right)_{K}$ & PROBABLY OK & OK, requires \\
$\sin 2 \beta$ & OK & non-trivial flavor physics \\
upper limits on nEDM, eEDM & very good & OK \\
baryon asymmetry & NO & OK \\
strong CP problem & NO & OK \\
& & $?$
\end{tabular}

In the $\mathrm{K}$ and $\mathrm{B}$ systems all supersymmetry ef-

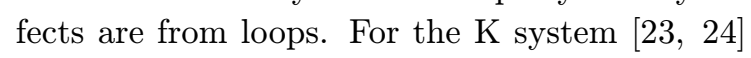
the dominant contributions are probably gluinosquark boxes and penguins. For $\Delta m_{K}$ the usual $\mathrm{SM}$ box is present, and the supersymmetry boxes could be of the same order or somewhat smaller - the hadronic uncertainties are large enough so one cannot tell. For $\varepsilon_{K}$ the imaginary part of the box is needed, so with our working assumption that $\delta_{C K M} \approx 0$ there is no SM contribution. The gluino-squark box is

The " $x$ " on a line means a squark L-R chirality flip, so it introduces a factor proportional to the off-diagonal elements of the trilinear couplings $A$. As we saw above, the gluino phase $\phi_{3}$ enters at 
the squark-gluino vertex - this is the same phase that can be studied at colliders. The contribution from this diagram is then proportional to

$$
\operatorname{Arg}\left(A_{s d} e^{-i \phi_{3}}\right)\left|A_{s d}\right|
$$

It is important [24] that the physical, reparameter ization-invariant phase combination $\phi_{s d}-\phi_{3}$ enters; the gluino phase alone would not give a meaningful result. The magnitude of the offdiagonal $s d$ element of the triscalar couplings must be of the right size to describe $\varepsilon_{K}$, so the supersymmetry approach can describe it but not explain it until there is a compelling flavor model. The SM gives a more natural description here, with the size of $\varepsilon_{K}$ given by the Jarlskog invariant, but recall we are pursuing $\mathrm{CP}$ violation beyond the SM because the SM fails when we get to the baryon asymmetry.

For $\varepsilon^{\prime} / \varepsilon$ the same sparticles enter in the penguin diagram,

which is again proportional to the same factor of Eq. 9.1, so the supersymmetry description is significantly constrained in order to describe both $\varepsilon$ and $\varepsilon^{\prime} / \varepsilon$. Results are usually presented in terms of quantities $\delta_{A B}^{f, i j}$, where $f$ and $i, j$ are flavor indices, and $A, B$ are chirality indices. We obtain a consistent description if

$$
\left|\delta_{L R}^{d, 12}\right| \approx 3 \times 10^{-3}, \quad \operatorname{Arg}\left(\delta_{L R}^{d, 12} M_{3}^{*}\right) \approx 10^{-2}(, 9.2)
$$

where the second quantity is proportional to the factor of Eq. 9.1.

An interesting thing to note here is that the size of the phase in Eq. 9.1 is perhaps reasonable because it can arise naturally in theories, where relations often occur among soft terms; such a result can indeed occur in the D-brane model described below. In that model $\phi_{\delta_{L R}^{d, 12}}=\phi_{A_{s d}}=$ $\phi_{M_{3}}$, so at the high scale $\operatorname{Arg}\left(\delta M_{3}^{*}\right)$ vanishes, and a small value is generated because $\phi_{A}$ and $\phi_{M_{3}}$ run differently.

Next turn to the B system [24]. Here all decays (except $b \rightarrow s \gamma$ ) have a tree level contribution, so (with the assumption that $\delta_{C K M} \approx 0$ ) we expect the $\mathrm{B}$ system to be superweak, i.e. all effects on the $\mathrm{B}$ system arise from mixing effects, $\varepsilon_{B}^{\prime} \approx 0$. The dominant mixing is generated by the chargino-stop box,

and the phases $\phi_{2}, \phi_{\mu}, \phi_{A_{t}}$ enter at the vertices. With our assumptions we predict $(\sin 2 \alpha$ and $\sin$ $2 \beta$ are defined to be the $\mathrm{CP}$ asymmetries measured in the decays $B_{d} \rightarrow \pi^{+} \pi^{-}$and $\psi K_{s}$ )

$$
\sin 2 \alpha=-\sin 2 \beta
$$

since the decay phase is zero. We also expect $|\sin \beta / \sin \alpha|=1$ (this does not uniquely follow from the previous equation, but in our model it does), while in the SM $|\sin \beta / \sin \alpha|=\left|V_{u b} / V_{c b} \sin \vartheta_{C}\right| \leq$ 0.45. Note that these results do not depend on any of the soft parameters, so they are general tests of the whole approach. Numerical studies show that there are regions of parameter space where this model gives a value of $\sin 2 \beta$ consistent with $\Delta m_{B_{d}}$ and recent measurements. If $\delta_{C K M}$ is not small we have to do a more detailed quantitative study, which is underway.

The CP asymmetry for $b \rightarrow s \gamma$ is particularly interesting because there is no tree level contribution. The SM CP asymmetry is very small [25], about half a percent. The supersymmetric loops can be of the same order as the SM loops (subject to the constraint that the $\mathrm{BR}$ is consistent with data), so a much larger CP asymmetry is possible. Simple estimates give several percent, and $10-15 \%$ is possible in special cases. This measurement is relatively clean since one is looking at a charge asymmetry, and many systematic problems cancel in ratios. It could be the first place in meson decays where physics beyond the SM is found. A $2 \%$ measurement would 
require perhaps 3000 events of each sign, which is reasonable for the $\mathrm{B}$ factories.

\section{BARYOGENESIS}

One of our most important clues to physics beyond the SM is the fact that the universe is made of baryons and not antibaryons. It is known that $\mathrm{CP}$ violation is a necessary condition to explain that asymmetry, and also that the SM cannot explain the baryon asymmetry value of $\delta_{C K M}$. Several approaches are known [26-6 that seem to be capable of explaining the baryon asymmetry, though in all cases the analysis is very complicated and the resulting numbers are still very uncertain. One attractive way to generate the baryon asymmetry as the universe cools is at the EW phase transition. Although such non-perturbative effects are very difficult to calculate, it is thought that the effects of EW sphalerons are strong enough to wash out any baryon asymmetry generated at higher temperatures, until the universe has cooled below the temperature at which the EW symmetry is broken. That leads many people to expect that the asymmetry generated at the EW phase transition is the relevant one.

Several other ways to generate the asymmetry could be relevant. One is the Affleck-Dine mechanism. Energy stored in scalar fields at high temperatures is radiated into particles whose decay into baryons is asymmetric. The second is leptogenesis from decay of heavy Majorana neutrinos, typically of mass of order $10^{11} \mathrm{GeV}$. In theories that conserve $\mathrm{B}-\mathrm{L}$, sphaleron interactions will generate a baryon asymmetry from the lepton number violating Majorana neutrino decay. In both cases it is very interesting to examine the origin of the needed CP violating phase. In the first it is likely to be connected to the supersymmetry soft phases since the scalars typically have flat potentials until the supersymmetry is broken. In the leptogenesis case the origin of the phase is seldom discussed. One can imagine that it occurs in the couplings of the Ma- jorana neutrinos in the superpotential, Yukawa couplings or higher dimension operators, or in soft terms involving the Majorana fields. Further work is needed to understand this important question. Another possibility is GUT baryogenesis preserved by B-L conservation and involving GUT Yukawa phases.

When the baryon asymmetry is generated at the EW phase transition, it involves the interactions of charginos, neutralinos, and stops inside and outside of growing bubbles where the EW symmetry is broken. If these sparticles are light enough, which essentially means lighter than $m_{t o p}$, an asymmetry will be generated. It will be proportional to quantities such as $\sin \left(\phi_{2}+\phi_{\mu}\right), \sin \left(\phi_{1}+\right.$ $\left.\phi_{\mu}\right)$, and other such phases including $\phi_{A_{t}}$. There are a number of uncertainties in the calculations, and perhaps the most difficult is how much of the asymmetry is left after all the sphaleron interactions become unimportant. In any case, learning the origin of the $\mathrm{CP}$-violating phase responsible for baryogenesis is a central issue in particle physics, with many possible implications from string theory to collider phenomenology and to cosmology.

\section{IS THE LSP THE COLD DARK MATTER?}

Let us assume that one day superpartners are found at colliders, and the LSP escapes the detectors. In addition, WIMP signals are seen in the underground detectors (DAMA, CDMS, and others), and perhaps in other large underground detectors and space-based detectors. Has the cold dark matter (CDM) of the universe been observed? Maybe, but those signals don't demon-

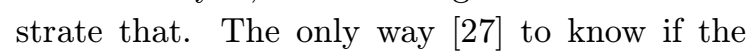
CDM has been detected is to calculate its contribution $\Omega_{L S P}$ to the relic density $\Omega$, and show that $\Omega_{L S P} \approx 0.3$. In fact, to some extent a large scattering cross section, which make direct detection easier, is correlated with a large annihilation cross section, which reduces the relic density. 
This subject is relevant in these lectures because the calculation of the relic density depends on knowing $\tan \beta$ and various soft parameters, including some of the phases. We expect the LSP to be the lightest neutralino (though the gravitino is a possibility, as are sneutrinos if the LSP relic density is small; in these lectures we assume conservation of R-parity or an equivalent quantum number). Recalling the discussion of neutralinos above, the lightest eigenvalue of the neutralino mass matrix will be a linear combination of the four neutralino symmetry eigenstates. The coefficients in the "wave function" that specifies the linear combination each depend on the entries in the neutralino mass matrix, $M_{1}, M_{2}, \mu, \phi_{1}+\phi_{\mu}, \phi_{2}+\phi_{\mu}$, and $\tan \beta$. Unless all of these are measured it is not possible to reliably calculate the relic density. The eigenvectors of the mass matrix determine the couplings of the LSP to particles that will appear in the annihilation diagrams. Additional soft parameters can enter. For example, the annihilation of LSP's that largely determines how many remain as the universe cools can proceed mainly through stau exchange, in which case the stau mass and its coupling to the LSP have to be measured. The phases can be very important, for several reasons. In addition to making it harder to measure $\tan \beta$, they can also cause explicit variation of $\Omega_{L S P}$ as we will see in the figures, and they can shift the normal P-wave suppressed annihilation to helicity-suppressed S-wave annihilation since they mix final states with different CP.

There are also other difficulties in interpreting any direct detection results, such as relating cross sections on nuclei to cross sections on protons and then quarks, figuring out the local galactic density of WIMPs and their velocity distributions, and so on. The issues raised here remain even if all these other questions are answered. Figure 11.1 [27] shows the region in the $\Omega_{L S P} h^{2}-\sigma_{p}$ plane where results could occur as various soft parameters are varied; $\sigma_{p}$ is the LSPproton scattering cross section, a useful measure of the strength of LSP interaction. A reference point is shown as a dark square. The allowed region if soft parameters are measured to $5 \%$ is grey, and the allowed region for $20 \%$ measurements is shown by the dots. If the soft parameters are measured to an accuracy of about $5 \%$ then $\Omega_{L S P}$ is determined by laboratory data to a good accuracy (the Hubble parameter $h$ will be accurately known by the time it is needed for this physics).

It would be very exciting if the CDM of the universe could be explained by particle physics laboratory data. This figure illustrates how direct detection (in the DAMA or CDMS or other experiments) does not determine $\Omega_{L S P}$ - for example, imagine a line across the figure at a "measured" value of $\sigma_{p}$. The relic density varies by a factor of five or more along such a line. For this figure the soft phases were set at $0, \pi$.

Figure 11.2 [277] shows how the relic density (the left side) and proton-LSP cross section (the right side, in units of $10^{-6} \mathrm{pb}$ ) vary with the phases relative to one reference point. This can introduce an additional factor of seven or so in the value of $\Omega_{L S P}$, so that the phases also must be measured to $5-10 \%$ accuracy before we can know the relic density even after the LSP is observed.

Figure 11.3 [2] $\left.\overline{2}_{1}\right]$ shows a scatter plot of the variation of $\Omega_{L S P}$ and the LSP mass as the phases are allowed to vary. The LSP mass is not very sensitive to the phases for the soft parameters used here. But imagine a vertical line at a given value of the LSP mass along which the relic density varies by a factor of about seven (remember that errors on the LSP mass will mean including the whole range shown). So measuring both the LSP mass and the LSP-proton cross section will still allow a factor of 5-10 variation in the relic density. Only measuring the soft parameters themselves, to a good accuracy, allows a meaningful determination of the relic density. As we will discuss below, the needed measurements of the soft parameters including the phases can only be done with data from a lepton collider with a polarized beam, and energy above the threshold for producing lighter charginos and neutralinos. 


\section{A STRING-MOTIVATED (D- BRANE) MODEL FOR THE SOFT PARAMETERS}

Now that we have looked at a number of examples, we want to return to the general structure of $\mathcal{L}_{\text {soft }}$. Although most of our considerations are quite general, it is always very important to have an explicit model in hand to test ideas and results. It is also important to have a model to illustrate how the many parameters of $\mathcal{L}_{\text {soft }}$ reduce to a few once there is theoretical structure. We also want a string based model since our ultimate goal is connecting the measured $\mathcal{L}_{\text {soft }}$ to string theory. We want a model where the phases are not forced to be zero, and gaugino masses are not forced to be degenerate. We want a realistic embedding of the SM so we can include EWSB, and no exotic particles already excluded by experiment. At the present time no models based on specific compactifications are satisfactory. The approach of Ibanez and collaborators

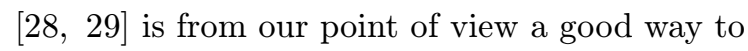
proceed. They introduce a fruitful parameterization of supersymmetry breaking (Eq. 12.1, 12.2), allowing the calculation of $\mathcal{L}_{\text {soft }}$ in particular string models. We [30in specifically use the results of ref. 29,30 .

Here we'll focus on orbifold compactifications of Type I string theory (which can be viewed as orientifold compactifications of Type IIB string theory). In such models with open (type I) string sectors, consistency conditions require the addition of D-branes, on which the open strings end. The number and type of D-branes depends on the chosen orientifold. In this approach it is assumed that supersymmetry is broken on other branes, and the breaking is transmitted to the SM branes by F-term vev's of closed string sector fields (the dilation $S$ and moduli $T_{i}$ ); the form of the F-term vev's of $S$ and $T_{i}$ is specified below. We can achieve our goals by using two intersecting 5-branes, as illustrated below:

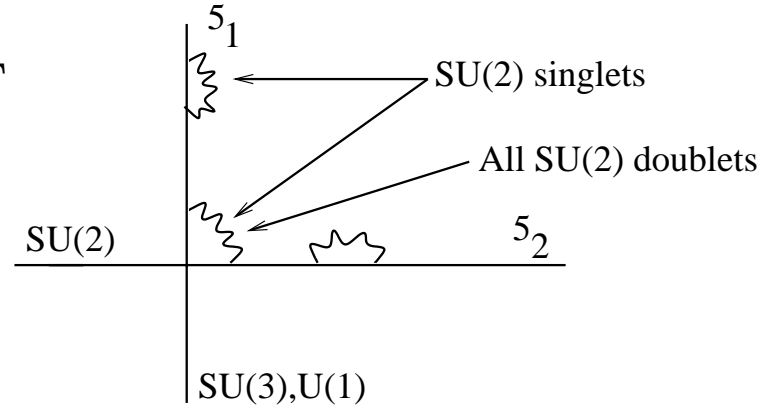

The strings representing the SM particles are massless modes and thus of zero length, but are shown for illustrative purposes in this figure. We choose to embed the SM gauge group such that $\mathrm{SU}(3)$ and $U(1)$ are associated with one of the intersecting branes, and the $\mathrm{SU}(2)$ is from the other. Thus all the doublets, quark and lepton and Higgs, must touch both branes at the intersection since they have both $\mathrm{SU}(2)$ and $\mathrm{U}(1)$ quantum numbers. The SU(2) singlets can be on either brane or possibly connecting them at the intersection. Given the basic approach and our goals as stated above this embedding is essentially unique. Other brane alternatives with the same embedding of the gauge groups are related to this by redefinitions and dualities. Following references 28 and 29 , the F-term vev's are taken to be

$$
\begin{gathered}
F^{S}=\sqrt{3}\left(S+S^{*}\right) m_{3 / 2} X_{0} e^{i \alpha_{S}} \\
F^{T_{i}}=\sqrt{3}\left(T_{i}+T_{i}^{*}\right) m_{3 / 2} X_{i} e^{i \alpha_{T_{i}}}
\end{gathered}
$$

The $X_{i}$ satisfy $\sum_{i=0}^{3} X_{i}^{2}=1$. Physically, the $X_{i}$ measure the relative contributions of the dilaton and moduli to the supersymmetry breaking. Given these F-term vev's one can calculate soft terms with the knowledge of the superpotential, Kahler potential, and gauge kinetic function $f_{\alpha}$ (in which $\alpha$ labels the gauge groups).

To illustrate how the soft terms arise it is worthwhile to write down two results. First, we express the superpotential and Kahler potential for the matter fields $\Phi$ in the theory in a general 
(string-motivated) form as $W=Y_{\alpha \beta \gamma} \Phi_{\alpha} \Phi_{\beta} \Phi_{\gamma}$, $K=K_{0}+\tilde{K}_{\alpha}\left|\Phi_{\alpha}\right|^{2}$ Given the parameterization of the F-term vev's above, the soft terms can be calculated using (standard) super-gravity techniques. Writing

$$
-\mathcal{L}_{\text {soft }}=\tilde{A}_{\alpha \beta \gamma} \Phi_{\alpha} \Phi_{\beta} \Phi_{\gamma}+\frac{1}{2} M_{a} \lambda_{a} \lambda_{a}
$$

the results for the soft triscalar couplings $\tilde{A}$ and gaugino masses $M_{a}$ are

$$
\begin{array}{r}
M_{1}=M_{3}=\sqrt{3} m_{3 / 2} X_{1} e(12.6) \\
M_{2}=\sqrt{3} m_{3 / 2} X_{2} e(12.7) \\
m_{Q_{a}}^{2}=m_{L_{a}}^{2}=m_{H_{u, d}}^{2}=m_{3 / 2}^{2}\left(1-\frac{3}{2}\left(X_{0}^{2}+X_{3}^{2} 2\right) 8\right) \\
m_{U_{1}}^{2}=m_{D_{1}}^{2}=m_{E_{1}}^{2}=m_{3 / 2}^{2}\left(1-3\left(X_{2}^{3}\right) 9\right) \\
m_{U_{2}}^{2}=m_{D_{2}}^{2}=m_{E_{2}}^{2}=m_{3 / 2}^{2}\left(1-\left(1 X_{3}^{2}\right) 0\right) \\
m_{U_{3}}^{2}=m_{D_{3}}^{2}=m_{E_{3}}^{2}=m_{3 / 2}^{2}\left(1-3\left(X_{2}^{2} .\right) 1\right)
\end{array}
$$

In general, the soft trilinear couplings have a hierarchical structure related to that of the Yukawa couplings, but are not directly proportional to them. In our case, the general form of the $\tilde{A}_{a, b}^{u, d, e}$

$$
\begin{gathered}
\tilde{A}_{\alpha \beta \gamma}=-\sum_{i=0}^{3}\left(\frac{\partial^{2} K_{0}}{\partial T_{i} \partial T_{i}^{*}}\right)^{-1 / 2}\left[\sum_{l=\alpha \beta \gamma} \frac{\partial \ln \tilde{K}_{l}}{\partial T_{i}}-\frac{\partial}{\partial T_{i}}-\frac{\partial K_{0}}{\partial T_{i}}\right] \times \\
Y_{\alpha \beta \gamma} \sqrt{3} m_{3 / 2} X_{i} e^{-i \alpha_{i}} \quad(12.4) \\
\tilde{A}^{u, d, e} \sim\left(\begin{array}{ccc}
0 & 0 & A_{u, d, e} Y_{13} \\
0 & 0 & A_{u, d, e} Y_{23} \\
0 & 0 & A_{u, d, e} Y_{33}
\end{array}\right)
\end{gathered}
$$

with the model giving $-A_{u, d, e}=M_{1}=M_{3}$. $M_{\alpha}=\frac{\sqrt{3} m_{3 / 2}}{2 R e f_{\alpha}}\left[\sum_{i=0,3} \frac{\partial f_{\alpha}}{\partial T_{i}}\left(\frac{\partial^{2} K_{0}}{\partial T_{i} \partial T_{i}^{*}}\right)^{-1 / 2} X_{i} e^{-i(1 i 2.5 \phi n \text { how the smaller elements of the Yukawa cou- }} \begin{array}{c}\text { Note that the smaller elements of } \tilde{A}_{u, d, e} \text { depend } \\ \text { plings are generated. }\end{array}\right.$

These results illustrate how the phases enter when the F-term vev's are complex, and also how if the Yukawa couplings are complex then it is natural for the soft trilinear couplings to be complex as well. The Yukawa couplings can in principle depend on the moduli. Here the $\tilde{A}_{\alpha \beta \gamma}$ are labeled by the scalar fields; these have to be rearranged into the $3 \times 3$ flavor matrix to compare with the usual form for the triscalar couplings.

Equations 12.4, 12.5 will not be used in the following, and we will not go into detail about their derivation. They are shown only to illustrate for the reader how Yukawa's and their phases, and the F-term vev's, can enter into determining the soft terms.

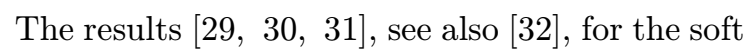
parameters in our example are
If instead of two 5-branes we had used a 9-brane and a 5-brane we would have a T-dual picture with all physical results the same, with the substitution of $X_{0} \rightarrow X_{3}$, etc. (see for example the discussion in $[\overline{2} \overline{9}]$ ).

The mechanism which provides the effective $\mu$ parameter in these models is not yet known definitively but is likely to be model-dependent, so for now it is better to simply parameterize it with a magnitude $\mu$ and phase $\phi_{\mu}$. At this stage the model depends on 8 parameters,

$m_{3 / 2}, \alpha_{2}-\alpha_{1}, X_{1}, X_{2}, X_{3}, \mu, \phi_{\mu}$, and t(ah2/3.3)

With this model, one can do all the usual collider and cold dark matter phenomenology, and also 
address aspects of $\mathrm{CP}$ violation physics and some flavor physics. The 105 parameters of the soft Lagrangian has collapsed down to this workable number. Once there is data the same kind of result is expected.

With our embedding gauge coupling unification is not automatic because the SM gauge groups are not all embedded on the same brane. If the real parts of the moduli vev's are all about the same size one will find approximate gauge coupling unification. Given the long-standing 10$15 \%$ discrepancy between the measured value of $\alpha_{3}$ and the value in the MSSM from naive running of the gauge couplings, this may actually be an advantage of this model, and may usefully constrain the parameters.

These kinds of models are attractive new ways to build less naive models that incorporate considerably more physics, both in the foundations and phenomenologically.

13. WHERE ARE THE SUPERPARTNERS AND HIGGS BOSON FINE TUNING?

Superpartners get mass from both the Higgs mechanism and supersymmetry breaking, the latter entering through the soft masses. For example, in the chargino mass matrix the off-diagonal elements come from the EW symmetry breaking when the Higgs fields get vev's, and the diagonal elements come from $\mathcal{L}_{\text {soft }}$. The EW contributions are typically of order $M_{W}$ or less. If the soft masses are large, the superpartner masses will generally be large.

There are four arguments that have been used to suggest that some superpartners will be light, i.e. near the EW scale. The strongest argument is from the success of supersymmetry in explaining the electroweak symmetry breaking. The EW symmetry breaking allows us to express $M_{Z}$ in terms of soft masses, so it gives one re- lation $\left[3 \overline{3}_{1}^{1}, \overline{3} \overline{4}_{1}, \overline{3} \overline{5}_{1}^{1}, \overline{3} \overline{6}\right]$ of the soft masses to a known mass. That alone is not enough to fix the soft masses, since several terms enter the relation (as we will discuss below), so one must impose some reasonable condition about cancellations among the contributions. There are no places in physics where large fine-tuning occurs or is acceptable once there is a theory, so it is appropriate to impose such a condition. What appears as fine-tuning is theory-dependent. The usual example is the precise equality of the electric charges of proton and electron, so atoms are neutral to a part in about $10^{20}$. If electric charge is quantized that is reasonable, if not it requires a huge fine-tuning. So we expect any acceptable theory to imply quantization of electric charge. Similarly, when we judge fine-tuning of soft masses we should do that in the presence of a theory that can relate the parameters. More generally, large cancellations only arise when they are exact, as for electric charge. For generic quantities in a theory there can be significant cancellations, but not very large ones. Even then, because parameters have different physical origins, and run differently from the unification scale to the weak scale, constraints remain. If supersymmetry is indeed the explanation for EWSB, then it is appropriate to impose reasonable finetuning constraints on the soft parameters. We will see that this implies gauginos and the Higgs boson should be light.

People have also argued [3근] that some superpartners, most likely sleptons, should be light or the LSP would annihilate too poorly and the large number of LSPs left would overclose the universe. But this assumes the LSP is the CDM - while I agree with that, it is an extra assumption. Further, there can be loopholes [3$\left.\overline{8}_{1}^{\prime}\right]$ from annihilation through a resonance or along particular directions in parameter space. A third argument is that if the baryon asymmetry is all or part from the EW phase transition it is neces-

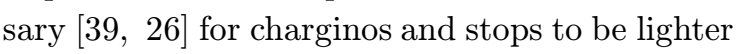
than about $m_{t o p}$, and for Higgs bosons to be fairly light. Again, that seems likely, but requires assuming the baryon asymmetry is indeed produced this way. Finally, light stops occur in 
most models because the stop mass matrix tends to have four elements of about the same size and therefore a small eigenvalue. While the reasons of this paragraph may indeed be relevant, the biggest success (as we discussed above) of supersymmetry is its explanation of the mechanism of the breaking of the electroweak symmetry, and we should be able to rely on the implications of that result.

The simplest way to write the resulting relation [36'] between $M_{Z}$ and the soft parameters is, for the SSM,

$M_{Z}^{2} \approx 7.2 M_{3}^{2}-0.24 M_{2}^{2}+0.014 M_{1}^{2}-1.8 \mu^{2}-1.4 M_{H}^{2}$

Here the soft parameters on the RHS are values at the unification scale, and the coefficients are determined by RGE running to apply at the weak scale. These numbers are for $\tan \beta=2.5$; for other values of $\tan \beta$ the coefficients change a little. The gluino mass is about $2.9 M_{3}$. Note that there is significant sensitivity to the gluino mass, and $\mu$, and less sensitivity to $M_{2}$ and $M_{1}$ or to the scalar masses. Details of the RGE running imply there is even less sensitivity to the scalar masses than is suggested by the coefficients here [401]. Clearly, if all of the parameters on the RHS are independent then several of them cannot be too large or supersymmetry is not actually explaining EWSB. But as we have discussed, in a real theory we expect relations among the soft parameters. Exact cancellations are not expected, since the parameters run differently down from the unification scale, and the coefficients depend on $\tan \beta$. Also, the physical origin of $\mu$ is likely to be different from the origin of the other soft masses so precise cancellations involving $\mu$ are unlikely.

The sensitivity to $M_{3}$ implies [3] 3 i the gluino cannot be too heavy if supersymmetry explains EWSB. Note that if one assumed degeneracy of the gaugino masses $M_{i}$ then one would also assume charginos and neutralinos must be very light, but that is only true if there is strict degeneracy. The chargino and neutralino masses are still constrained to be fairly light because they also depend on $\mu$, as the figures below show. We [36 $\left.{ }^{3}\right]$ have studied how heavy the gaugino and Higgs masses can be if supersymmetry does explain the EWSB even in a theory with cancellations, by using the Dbrane model of section 12 which allows significant cancellations. The results are shown in Figures 13.A, 13.B. The grey bands show the allowed regions. The curves of each type are for $\tan \beta=$ $2,3,10$. The amount of fine tuning is measured by the quantities $\Delta_{a}$, for a parameter $a$, where $\Delta_{a}=\left|\frac{a}{M_{Z}^{2}} \frac{\partial M_{Z}^{2}}{\partial a}\right|$. The vertical axis in the figures is the largest $\Delta_{a}$. In the bands the other soft parameters are varied. For these figures the phases are set to $0, \pi$ since there is not too much effect foromm $m_{\text {rartying }}^{2}$ thlem, except at small fine tuning where the cancellations are strong.

Sometimes people are uncomfortable about choosing an upper limit on the amount of fine-tuning, but if supersymmetry really is the explanation for the EWSB then there really cannot be much fine tuning. In that case, we can see that gluinos cannot be heavier than about $500 \mathrm{GeV}$, which is accessible at the Tevatron for most decay chains, the lighter chargino cannot be heavier than about $250 \mathrm{GeV}$, again accessible at the Tevatron, the LSP cannot be heavier than about $100 \mathrm{GeV}$ and the second neutralino cannot be heavier than about $250 \mathrm{GeV}$, so the sum of their masses is always accessible at the Tevatron. The Higgs boson cannot be heavier than about $105 \mathrm{GeV}$ (but remember that the current limits from LEP are only about $85 \mathrm{GeV}$ when evaluated correctly as explained in Section 7). Note that the significant constraints on superpartner masses apply only to gauginos and Higgs, not to squarks and sleptons [40"].

If supersymmetry really is the explanation of the electroweak symmetry breaking then superpartners will be produced at the Tevatron. 


\section{APPLICATION - WHAT EN- ERGY LEPTON COLLIDER IS SAFE TO BE CONFIDENT IT CAN DO SIGNIFICANT NEW PHYSICS EVEN AFTER THE TEVATRON AND LHC?}

If there were no limit to available resources we would not have to discuss questions such as this. A multi-TeV lepton collider with a polarized beam could clearly do important new physics even after LHC. But such a collider may not yet be technically feasible, and is certainly too expensive to build for many years. On the other hand, it is known how to build a 500-600 GeV $e^{+} e^{-}$ collider now, and (although there is no official cost estimates for such a collider) experts I have confidence in tell me that such a collider could be proposed and built beginning now, for a cost that appears to be reasonable given funding realities. But is such a collider, at most 3 times the LEP energy and considerably less than the LHC energy, worth building for the physics results it can provide after the Tevatron and LHC have taken data? In the best case such a collider could be taking data in 2010. The answer is yes, given reasonable guarantees (which have not yet been made precise) about luminosity and polarization. That is, a $600 \mathrm{GeV}$ linear electron collider is as safe a bet as one can make in physics for a facility that will provide major

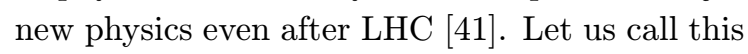
collider the $\mathrm{N}$ (ext) $\mathrm{P}$ (olarized)L(epton) $\mathrm{C}$ (ollider). The energy $600 \mathrm{GeV}$ emerges as a safe upper limit independently from both the Higgs sector and the SUSY sector, so it would be good if an initial (say) $500 \mathrm{GeV}$ collider were extendable to about $600 \mathrm{GeV}$.

The reasons why such an energy is safe comes in two parts. One involves the Higgs sector. The other involves supersymmetry, and depends strongly on understanding the soft-breaking Lagrangian, so it is a relevant application for these lectures.

First consider the Higgs sector argument briefly
[42] There is now a well-established upper limit on $M_{h}$ in the SM, from precision measurements, $M_{h} \lesssim 200 \mathrm{GeV}\left[4 \overline{3}_{3}^{\prime}\right]$. If a light Higgs boson in fact exists the bound is satisfied and NPLC can study it, and make a number of significant measurements that cannot be made at the Tevatron or LHC, such as the branching ratios to up-type quarks and to gluons. Even more importantly, if there is not a light Higgs boson but other physics that also contributes to the precision data in just the right way to fake the upper limit, then the tight limit plus good understanding of the theory allows [42 scalar state that can be studied at NPLC with a mass of at most $500 \mathrm{GeV}$ (500 is an upper limit - usually its mass is less. So to find this scalar probably $500 \mathrm{GeV}$ is enough, but $600 \mathrm{GeV}$ is safe. Further, (b) there must always be an associated piece of physics that affects the precision data in just such a way as to compensate for the effects of the heavier scalar. This additional contribution, a heavy fermion or Kaluza-Klein states, or something else, will also have effects that are detectable at NPLC (that may require running NPLC at the $\mathrm{Z}$ mass, but such running is expected at any linear electron collider if it is scientifically justified). Thus in the Higgs sector NPLC600 is a very good facility to have if there is indeed a light Higgs boson as is expected, and it is even better if there is no light Higgs. This argument provides strong justification for such a collider.

Next turn to the physics justification based on supersymmetry. We have seen in section 13 that if supersymmetry is indeed the explanation for the fundamental question of how the electroweak symmetry is broken so that particles can have mass in the Standard Model in a consistent way, then some superpartners (in particular, charginos and neutralinos) are not too heavy. In fact, at least the lightest chargino and the lighter two neutralinos could be studied at NPLC600. (Perhaps the light stop and sleptons could also be studied at NPLC600.) This is extremely important because it allows the measurement of $\tan \beta$ and the soft phases, which could not be done at LHC. 
To clarify this very important point, let us elaborate on what NPLC600 would be able to achieve that hadron colliders could not. There are four such physics opportunities in the supersymmetry sector. The first three are important, and the fourth is by far the most important. First, if nature is not supersymmetric at the weak scale it is essential to know that so we can focus on other directions. Knowing that would have a major effect on string theory activities as well. If signals for supersymmetry are not found at the Tevatron and LHC it will be hard to still think that low energy supersymmetry is part of nature, but because of the large backgrounds it is possible to hide supersymmetry signals at hadron colliders. Only a lepton collider above the threshold for some superpartners can be definitive here. Why should $600 \mathrm{GeV}$ be above that threshold? To repeat, if supersymmetry does explain the EW symmetry breaking we saw above that $600 \mathrm{GeV}$ should be a safe upper limit. If supersymmetry does not explain the EWSB it is unlikely to be part of the low energy description of nature.

Second, it is important to be able to demonstrate [44] that a set of signals is indeed supersymmetry. The spins of the superpartner candidates need to be measured. Many relations among coupling strengths predicted by supersymmetry need to be tested. For example, the quadratic Higgs coupling $\lambda$ should be given by gauge couplings $\left(g_{1}^{2}+g_{2}^{2}\right) / 2$ the $\tilde{g} \tilde{g} g$ coupling should be the same as the gluon self coupling $g_{3}$, the $\tilde{d} d \tilde{\gamma}$ coupling should be $\frac{1}{3} e$, the neutralinos and gluino should be Majorana particles, and so on. It is extremely hard to demonstrate any of these results at a hadron collider, while it is doable at NPLC600. Third, some superpartners or some BR of some superpartners or Higgs bosons may be invisible at hadron colliders, but detectable at NPLC600. That can happen if masses are close to degenerate, if some decays are invisible such as $h \rightarrow L S P+L S P$ (or $h \rightarrow$ Kaluza Klein modes or some other exotic channel), $\tilde{N} \rightarrow \tilde{\nu} \nu$, and so on. Various chargino, neutralino, Higgs, and stop modes can be in this category.

Finally we consider the most important point.
Let us assume that superpartners are indeed observed. As we have seen in these lectures, the central problem of particle physics is then to measure and understand the soft-breaking supersymmetry Lagrangian (and $\tan \beta$ ), and to use it to help formulate and test string theory. We have seen in these lectures that it is essential to learn the patterns of $\mathcal{L}_{\text {soft }}$, whether the soft masses are degenerate, whether some or all phases are large or not, and also the numerical values of the soft parameters. These quantities are at least as fundamental as the quark masses and the CKM mixing angles and phase.

Learning the quark masses and mixing angles did not lead to a breakthrough in understanding. Most likely that is because at the level of the string theory some Yukawa couplings are of order the gauge couplings and others are zero. Then those that are zero get small non-zero values as some symmetries are broken, and/or from non-perturbative operators and loop corrections. It may be that we have to understand a great deal of the theory before they can be explained. It is likely that the situation with the soft parameters will be simpler since all the superpartners get mass from them, so the leading effects will be important, and they will tell us explicitly about how supersymmetry is broken, and about compactification.

To measure the soft parameters it is necessary to have enough observables. Think about hadron colliders. There is much that hadron colliders will do well, such as measure the dominant quantum numbers of the LSP and therefore largely determined how supersymmetry breaking is mediated, and measure the mass of the LSP [1] is relevant for learning whether the LSP is the cold dark matter. One can measure the masses of most (though probably not all) of the superpartners. We have seen that at most the gluino (approximately) and gravitino (if it is the LSP) masses are directly connected to those in the Lagrangian; for the rest one must invert equations relating the Lagrangian parameters to the masses and other soft parameters including the phases and $\tan \beta$, as in the chargino example of Section 
5 or the Higgs example of Section 7. One can measure $\sigma \times B R$ for most superpartners, though certainly not all since the backgrounds are large and many signatures overlap. Since there are 33 superpartner and Higgs states, this could give 66 observables, though that is surely an overestimate, considerably fewer than the number of parameters. If one could measure differential cross sections in bins with significant statistics one would of course have more observables. But in practice that is probably not possible even at electron colliders usually only forwardbackward asymmetries are measured. So the total number of observables at hadron colliders is very unlikely to be enough to invert and solve for the soft parameters and $\tan \beta$. Possibly we will be lucky and the masses and BR will have values that allow some limited sector to be solved for its soft parameters, but we cannot count on that. (There has been some work reported on learning supersymmetry parameters at LHC, but always in the context of models assumed to be known, and with a number of assumptions about values of most parameters. Once there is data, of course experimenters will want to measure parameters without such assumptions.)

Lepton colliders are often characterized as being cleaner than hadron colliders, and that is true, but that is not the main reason they are important for the future of particle physics. The main reason is that they allow the measurement of more observables. The polarized beam immediately doubles the number of observables. Equally important, running at a second energy again doubles the number of observables, so effectively there are four times as many observables at a polarized lepton collider as at a hadron collider! Of course that is only true for the superpartners that are produced at the lepton collider, so some observables are lost because the relevant masses are too large. To get the full picture it is essential to have both the lepton collider and the hadron colliders. In fact, both LHC and the Tevatron are essential because the Tevatron produces the lighter superpartners and can tell us about their masses and $\mathrm{BR}$, allowing us to then untangle the complicated situation at the LHC where many superpartners will be produced and the heavier ones will have complicated decay chains through several intermediate steps. The Tevatron is complimentary to both LHC and NPLC600, to the latter in part because it produces colored states. Of course, finally results from low energy experiments such as $\mathrm{CP}$ violation at b-factories, EDMs, rare decays, proton decay, cold dark matter and more will be combined with collider data to fully learn the soft Lagrangian.

There are no realistic studies of how many observables NPLC600 can measure. Simulations under conditions where no favorable assumptions about decay BR are made, and with reasonable experimental errors and statistics included, are essential to confirm the qualitative analysis above. But given the existence of the Tevatron and LHC and b-factories and serious efforts on the other low energy experiments, it is likely that NPLC600 is both necessary and sufficient to let us measure enough about the world to proceed to formulate and test string theory.

\section{ACKNOWLEDGEMENTS}

I am grateful to M.Brhlik, D.Chung, K.Dienes, S.King, S.Mrenna, L.-T.Wang, and J.Wells for discussions and suggestions, and I particularly appreciate discussions, suggestions and contributions to these lectures from L.Everett.

\section{References}

[1] S.Dimopoulos and H.Georgi, Nucl. Phys. B193(1981)150; L.Girardello and M.Grisaru, Nucl. Phys. B194(1982)65.

[2] D.R.T.Jones, L.Mezincescu, and Y.P.Yao, Phys. Lett. B148(1984)317; L.J.Hall and L.Randall, Phys. Rev. Lett. 65(1990)2939; I.Jack and D.R.T.Jones, Phys. Lett. B457(1999)101; L.Diaz-Cruz, hep-ph/9906330.

[3] For a review, see the lectures of A.Masiero at this school. 
[4] S.Dimopolouos and D.Sutter, Nucl. Phys. B452(1995)496; H.Haber, proceedings of supersymmetry 1997, Nucl. Phys. Proc. Suppl. 62(1998)469.

[5] M.Brhlik and G.L.Kane, Phys.Lett. B437(1998)331.

[6] T.Ibrahim and P.Nath, Phys. Rev. D58(1998)111301; Phys. Rev. D57(1998)478; Phys. Lett. B418(1998)98.

[7] M.Brhlik, G.Good, and G.L.Kane, Phys. Rev. D59(1999)115004.

[8] M.Dugan, B.Grinstein, and L.Hall, Nucl. Phys. B255(1985) 413.

[9] S.Pokorski, J.Rosiek, and C.Savoy, hepph/9906206.

[10] M.Brhlik, L.Everett, G.L.Kane, and J.Lykken, Phys. Rev. Lett. 83(1999)2124; hep$\mathrm{ph} / 9908326$.

[11] M.B.Gavela, P.Hernandez, J.Orloff, O.Pene and C.Quimbay, Mod. Phys. Lett. A9(1994)795, Nucl. Phys. B430(1994)382; P.Huet and E.Sather, Phys. Rev. D51(1995)379.

[12] J.-M.Frere and M.B.Gavela, Phys. Lett. B132(1983); C.S.Lim, Phys. Lett. B256(1991)233; M.Dine, R.G.Leigh and D.MacIntire, Phys. Rev. Lett. B69(1992)2030; K.Choi, D.Kaplan and A.Nelson, Nucl. Phys. B391(1993)515; T.Kobayshi and C.S.Lim, Phys. Lett. B343(1995)122; J.-M.Frere and S.Abel, Phys. Rev. D55(1997)1623.

[13] For a different view, see arguments by D.Bailen et al, Nucl. Phys. B518(1998)92; Phys. Lett. B435 (1998)323; but see also J. Giedt, hepph/0007193 who presents examples that a CKM phase often does not arise even in the presence of complex moduli vev's.

[14] S.Martin and M.Vaughn, Phys. Rev. D509(1994)2282.

[15] A number of studies have examined this. See, for example, S.Y.Choi, A.Djouadi, M.Guchait, J.Kalinowski, H.S.Song, and P.Zerwas, hep$\mathrm{ph} / 0002023$, and refrences there in.

[16] A.Pilaftsis, Phys. Lett. B435(1998)88; Phys. Rev. D58(1998)096010.

[17] K.S.Babu, C.Kolda, J.March-Russell, and F.Wilczek, Phys. Rev. D59(1999)016004; D.A.Demin, Phys. Rev. D60(1999)055006; Phys. Lett. B456(1999)177; S.Y.Choi, M.Drees, and J.S.Lee, hep-ph/0002287; B.Grazdkowski,
J.Gunion, and J.Kalinowski, hep-ph/0001093; M.Carena, J.Ellis, A.Pilaftsis, and C.Wagner, hep-ph/0003180.

[18] G.L.Kane and Lian-Tao Wang, hepph/0003198, to appear in Phys. Lett.

[19] S.Mrenna, G.L.Kane, and Lian-Tao Wang, hepph/9910477, to appear in Phys. Lett.

[20] S.Dimopoulos and S.Thomas, Nucl. Phys. B465(1996)23.

[21] A.Strominger and E.Witten, Commun. Math. Phys. 101(1985)341.

[22] M.Dine, R.G.Leigh, and D.MacIntire, hep$\mathrm{ph} / 9307152$.

[23] Some of a number of recent refrences are A.Masiero and H.Murayama, Phys. Lett. 83(1999)9107; S.Khalil, T.Kobayashi, and A.Masiero, Phys. Rev. Lett. D60(1999)075003; R.Barbieri et al hep-ph/9908255; A. Buras et al, hep-ph/9908371; G.Eyal et al, hepph/9908382; D.Demin, A.Masiero, and O.Vives, hep-ph/9909325, hep-ph/0011337; A.Kagan and M.Neubert, hep-ph/9908404; Y.Grossman et al, hep-ph/9909297.

[24] M.Brhlik, L.Everett, G.L.Kane, S.F.King, and O.Lebedev, Phys. Rev. Lett. 84(2000)3041.

[25] A.Kagan and M.Neubert, Phys. Rev. D58(1998)094012.

[26] Some recent articles from which the literature can be traced are A.Dolgov, Phys. Rev. 222C(1992)309; "Recent progress in Affleck-Dine Baryogenesis", by K.Engvist, hepph/0002125; "Baryogenesis Through Leptogenesis", R.Barbieri et al, hep-ph/9911315; A.Riotto and M.Trodden, Ann. Rev. Nucl. Sci. 49(1999)35 hep-ph/9901362; M.Brhlik, G.Good, and G.L.Kane, hep-ph/9911243.

[27] M.Brhlik, D.J.Chung, and G.L.Kane, hep$\mathrm{ph} / 0005158$.

[28] See A.Brignole, L.Ibanez, and C.Munoz, hepph/9707209, in Perspectives on Supersymmetry, and G.L.Kane, World Scientific 1998, for earlier work on this approach..

[29] L.Ibanez, C.Munoz, and S.Rigolin, hep$\mathrm{ph} / 9812397$.

[30] M.Brhlik, L.Everett, G.L.Kane, and J.Lykken, Phys. Rev. Lett. 83(1999)2124, hepph/9905215; hep-ph/9908326.

[31] L.Everett, G.L.Kane and S.F.King, hepph/0005204, to appear in JHEP. 
[32] S.Khalil, T.Kobayashi, and O.Vives, Nucl. Phys. B580(2000)275.

[33] J.Ellis, K.Enqvist, D.Nanopoulos, and F.Zwirner, Nucl. Phys. B276(1986)14; R.Barbieri and G.Giudice, Nucl. Phys. B306(1998)63.

[34] G.W.Anderson and D.J.Castano, Phys. Lett. B347(1995)300; S.Dimopoulos and G.Giudice, Phys. Lett. B357(1995)573; P.Chankowski, J.Ellis, and S.Pokorski, Phys. Lett. B423(1998)327; R.Barbieri and A.Strumia, Phys. lett. B433(1998)63; K.L.Chan, U. Chattopadhyay, and P. Nath, Phys. Rev. 58(1998)096004.

[35] D.Wright, hep-ph/9801449.

[36] G.L.Kane and S.F.King, Phys. Lett. B451(1999)113, hep-ph/9810374; M.BasteroGil, G.L.Kane and S.F.King, hep-ph/9910506, Phys. Lett. B474(2000)103.

[37] See L.Roszkowski, Phys. Lett. B278(1992)147; J.D.Wells, Phys. lett. B443(1998)196; J.Ellis, T.Falk, G.Ganis, and K.Olive, hep-ph/0004169.

[38] M.Drees and M.Nojiri, Phys. Rev. D47(1993)376; J.Feng, K.Matchev, and F.Wilczek, Phys. lett. B482(2000)388.

[39] See, for example, M.Carena, M.Quiros, and C.Wagner, Nucl. Phys. B524(1998)3.

[40] For a recent study of this result, see J.Feng, K.Matchev, and T.Moroi, Phys. Rev. D61(2000)075005.

[41] See my talks at the NLC meeting, Berkeley 2000, March 2000, www-physics.lbl.gov/divoffice/berkeley2000.html, and at susy2000.

[42] G.L.Kane and James D.Wells, hep-ph/0003249.

[43] See the LEP EW Working Group, www.cern.ch/LEPEWWG/welcome.html.

[44] A number of such tests have been studied at European, Japanese, and American linear collider workshops and in associated papers. Much of the literature can be traced from H.Murayana and M.Peskin, Ann Rev. Nucl. Sci. 46(1996)533. 